\title{
Laboratory Evaluation of the Marking Effect of Sudan Red 7B on Subterranean Termites (Reticulitermes speratus) in Republic of Korea ${ }^{1}$
}

\author{
Ik-Gyun $\mathrm{IM}^{3,4} \cdot$ Gyu-Seong HAN(D ${ }^{2,4, \dagger}$
}

\begin{abstract}
The dye marker Sudan Red 7B was tested as an effective and appropriate marking method for searching the territory of the termite, Reticulitermes speratus, in Korea. The sensitivity of termites to the dye marker was evaluated by measuring the feed consumption amount, survival rate, retention time, and transfer efficiency of $R$. speratus according to the concentration and feeding period of Sudan Red 7B. As the concentration conditions of dye marker increase, Sudan Red 7B remarkably reduced the feed consumption of the termites. The survival rate was not significantly different from that of the control group (more than 90\%), except when feed containing $1 \%$ or $2 \%$ concentrations of the dye marker was given for five days. The duration of the marking effect persisted for seven days, even at a concentration of $2 \%$ dye marker in the feed. No dye transfer by trophallaxis occurred between termites marked with Sudan Red $7 \mathrm{~B}$ and unmarked termites.
\end{abstract}

Keywords: termite, Reticulitermes speratus, marking, termite monitoring, termite territory

\section{INTRODUCTION}

The representative termite (Reticulitermes speratus) living in Korea is a subterranean termite, which inflicts structural and aesthetic damage by accessing wooden structures and wooden cultural heritages through the ground. During the renovation of Treasure No. 1742, Jeongjagak in Sungneung, Donggureung (Guri-si, Gyeonggi-do), in 2019, damage from termites was confirmed on the walls and top. Accordingly, it was decided to dismantle and repair the entire Jeongjagak in order to repair the deteriorated and dam- aged members of the entire building (Cultural Properties Committee, 2019).

Currently, regular monitoring is being conducted on important wooden cultural properties in Korea to diagnose damage caused by termite infestation(Cultural Heritage Administration, Cultural Heritage Care Support Project, 2019; Chung et al., 2015; Kim and Han, 2019; Kim et al., 2017; National Research Institute of Cultural Heritage, 2020). The National Research Institute of Cultural Heritage conducts annual diagnosis of termite habitat status using termite detection dogs and investigations of chewing marks through visual ob-

\footnotetext{
${ }^{1}$ Date Received August 17, 2020, Date Accepted September 15, 2020

2 Department of Wood and Paper Science, Chungbuk National University, Cheongju 28644, Republic of Korea

${ }^{3}$ Department of Cultural Heritage Science, Chungbuk National University, Cheongju 28644, Republic of Korea

${ }^{4}$ Heritage Science Center, Chungbuk National University, Cheongju 28644, Republic of Korea

$\dagger$ Corresponding author: Gyu-Seong HAN (e-mail: wood@chungbuk.ac.kr, ORCID: 0000-0003-3835-2063)
} 
servation for national treasures, treasures, national folk cultural properties, and historic sites (National Research Institute of Cultural Heritage, 2020). In addition, the Cultural Heritage Care Project Group regularly monitors the damage caused by termites through the installation of wood elements, diagnosis of invasion and attack using microwave detection devices and investigation of chewing marks through visual observation for wooden buildings among regional wooden cultural heritages (Cultural Heritage Administration, Cultural Heritage Care Support Project, 2019). As of 2019, a total of 23 cultural property care projects are active in 17 provinces nationwide. non-destructive techniques such as ultrasonic waves, portable microscopes, and temperature monitoring were performed on wooden structures attacked by termites (Son and Lee, 2007, 2008).

However, Subterranean Termite has a habit of moving by forming networks in the ground (King and Spink, 1969). In the case of an urban area, it is very difficult to investigate the size of the colony and the extent of its activity area because it is impossible to observe with visual observation because the passage is extended under a paved road in contact with the ground or with wood elements (Grace et al., 1990). As a result of the research on the excavation of the Reticulitermes speratus tunnel, it has been confirmed that it is possible to move actively in both plane and vertical direction (Hwang and Lee, 2010). It is possible to check whether there is any visible termite damage through monitoring of termite invasion and infestation on wooden cultural properties currently conducted in Korea. However, detailed information on the extent of attack to wooden structures by termite colonies moving and invading through the ground or changes in the seasonal activity area of termites cannot be obtained.

For termite control, it is very important to determine the size and activity area of termite colonies. Accord- ingly, studies on the identification and marking characteristics of the foraging territory of termites have been conducted overseas since the 1970s using a dye marker (Nobre et al., 2007; Su and Scheffrahn, 1986, 1988; Su et al., 1991, 1993; Grace et al., 1989; Tsunoda et al., 1998, 1999; Thorne et al., 1996). As a reagent applied for termite marking, Sudan Red 7B (Su et al., 1983; Su, 1988; Grace and Abdallay, 1989, 1990 ) is representative. In addition, various color-specific dye markers such as Nile Blue A, Neutral Red, and Fast Green were applied to confirm survival rate, and marking persistence(Atkinson, 2004; Sattar et al., 2007, Xing et al., 2013). Thorne et al. (1996) pointed out that, the conventional test (filter paper diet) for persistence of a vital dye is not a realistic approximation of a field diet or exercise regime, and should be reevaluated. Accordingly, general food, not filter paper, was fed as a food to check retention time of Sudan Red 7B in this study.

As described above, termite marking using a dye marker is considered to be a very important part for termite control and monitoring in that it can confirm various activity patterns and ecological characteristics of the target termite colony. However, in Korea, research related to this remains very rudimentary.

Therefore, in this study, we tried to find an effective and appropriate marking method to search the realistic approximation of the termite (Reticulitermes speratus) colony territory living in Korea. Sudan Red 7B was applied as a dye marker for termites collected near Sejong City. The sensitivity of termites to the dye marker was evaluated by measuring the feeding amount, survival rate, retention time and trophallaxis of Reticulitermes speratus according to the concentration and feeding period of Sudan Red 7B. Through this study, we propose an effective and appropriate method for evaluating the marking effect in the search for Reticulitermes speratus colonies. 


\section{MATERIALS and METHODS}

\subsection{Materials}

\subsubsection{Termite}

Wood attacked by termites was collected from the hills in the Goundong area of Sejong-si and stored in a storage container in the laboratory. Termite individuals to be used in the experiment were separated as follows by modifying the method of Judd and Fasnacht (2007), Tamashiro et al. (1973). Immediately before the start of the experiment, corrugated cardboard was placed in the storage container for a certain period of time so that termites were transferred to the corrugated cardboard, so that termites could be separated without decomposition of the wood in the container. The termite used in the experiment was identified as Reticulitermes speratus.

\subsubsection{Dye marker}

As a dye marker for termites, Sudan Red 7B, a red dye applied to Coptotermes formosanus and Reticulitermes flavipes, was used in previous studies abroad. Sudan Red 7B was used without purification as a reagent grade. Using acetone as a solvent, 0.5\%, 1.0\% and $2.0 \%$ diluents were manufactured.

\subsubsection{Silica sand}

Silica sand (Silica sand No 7, Saeron Industry, Korea) with relatively small particles was used as maintain humidity for the termite trial arena. After washing three times with $97 \%$ ethanol to remove impurities from silica sand, it was washed three times with distilled water. After that, large particles were removed by filtering through a 50-300 $\mu \mathrm{m}$ standard mesh. Finally, it was used after drying for 3 days at $60^{\circ} \mathrm{C}$ in a dry oven.

\subsection{Methods}

\subsubsection{Marking}

The sample for termite marking was prepared as follows. Filter paper with a diameter of $90 \mathrm{~mm}$ (Advantec No2, Toyo Roshi Kaisha LTD., Japan) was divided into 4 equal parts (about $1.9 \mathrm{~g}$ ) and dried in a dry oven at $60^{\circ} \mathrm{C}$ until constant weight. $200 \mu \mathrm{l}$ of the dye marker prepared for each concentration condition was applied to the dried filter paper, and after volatilizing acetone, it was dried again to a constant weight in a dry oven at $60^{\circ} \mathrm{C}$. As the dye marker used in the experiment, Sudan Red 7B was diluted with acetone at concentrations of $0.5 \%, 1.0 \%$, and $2.0 \%$ (wt/wt). As a result, the weight ratio of the dye marker contained in the feed sample was $0.05 \%, 0.1 \%$, and $0.2 \%$ for each concentration, respectively.

The trial arena for termite marking was manufactured as follows (Fig. 1). The pretreated silica sand and distilled water were mixed in a ratio of $50 \mathrm{~g}$ : 5 $\mathrm{g}(\mathrm{wt} / \mathrm{wt})$ and placed $90 \mathrm{~mm}$ petri dish. A sample with containing dye marker was placed top of silica sand containing distilled water in the $90 \mathrm{~mm}$ petri dish. 200 workers were added to each petri dish to enable

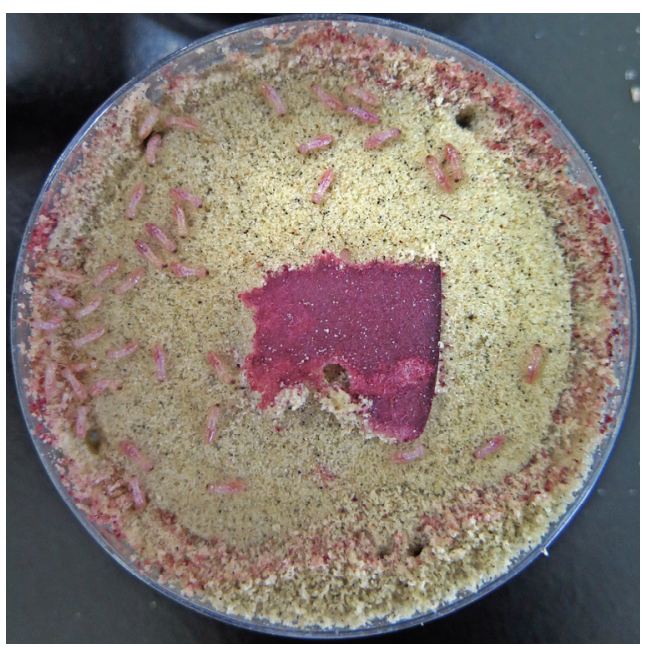

Fig. 1. The trial arena for termite marking. 
feeding activities. The termites were exposed under No- Choice conditions for 3 days and 5 days according to the concentration of the dye marker, so that the workers were stained with the dye marker. Each experiment was carried out in a total of 15 replicates, 3 times and 5 repetitions for each condition.

\subsubsection{Feed consumption}

For feed samples (filter papers) impregnated with different concentrations $(0.5 \%, 1 \%$, and $2 \%)$ of the dye marker, the feeding amounts according to the feeding periods of termites were measured. After each feeding period, filter papers were taken out, dried to a constant weight at $60^{\circ} \mathrm{C}$ in a dryer, and the weights were measured and compared with the weights of the samples before feeding.

\subsubsection{Survival rate}

In order to check the survival rate of termites shown in 2.2.1, the following food was fed for 5 days and 10 days. The feed source used to investigate the survival rate of the marked workers was prepared by combining soil and wood flour according to the recipe prepared by laboratory. In a feeding activity vial having a diameter of $40 \mathrm{~mm}$ and a height of $100 \mathrm{~mm}$, $40 \mathrm{~g}$ of a feed source and distilled water were mixed in a ratio of 3:1 and placed. $15 \mathrm{ml}$ of agar at a concentration of $3 \%$ was injected to prevent evaporation of moisture from the feed source and at the same time to suppress microbial germination on the feed source surface as much as possible. According to 2.2.1, 200 marked workers for each condition were divided into 100 individuals and placed in a trial vial. The number of replicates was 20 for each condition, and after 5 and 10 days, 10 replicates for each condition were randomly selected to check the survival rate.

\subsubsection{Retention time of dye marker}

The retention time of the dye marker was checked for termites fed with sample containing $2 \%$ concentration of the dye marker. The termites fed with sample containing the dye marker for 5 days were observed with an stereoscopic microscope for marking persistence over time for 1,4 , and 7 days while feeding general food.

\subsubsection{Transfer effect of dye marker between termites}

In the same colony, it was confirmed whether staining transfer by the marked reagent occurred between the marked and unmarked individuals. 50 marked workers and 50 unmarked workers were introduced to the same petri dish. The marked units at a concentration of $2 \%$ for 5 days were targeted. At this time, filter paper impregnated with $200 \mu \mathrm{l}$ of distilled water was put as a feed source on the petri dish. After 48 hours, the number of marked individuals was measured to confirm the transfer effect of the dye marker.

\section{RESULTS and DISCUSSION}

\subsection{Feed consumption according to the concentration of the dye marker}

When Sudan Red 7B was used as the dye marker, the results of the food intake of termites according to the concentration of the dye marker $(0.5 \%, 1.0 \%$, $2.0 \%$ ) and feeding period (3 days, 5 days) are shown in Fig. 2 and Table 1.

It was confirmed that the feeding amount for 5 days was higher than that for 3 days in the control and all concentration conditions. Because the longer the food exposure time the greater the amount of food that surviving termites feed on. When Sudan Red 7B, a dye marker, was added to the feed, the feed consumption was significantly reduced. Compared to the feeding capacity of the control group, the same results were indicated in two consumption period conditions: 
Laboratory Evaluation of the Marking Effect of Sudan Red 7B on Subterranean Termites (Reticulitermes speratus) in Republic of Korea

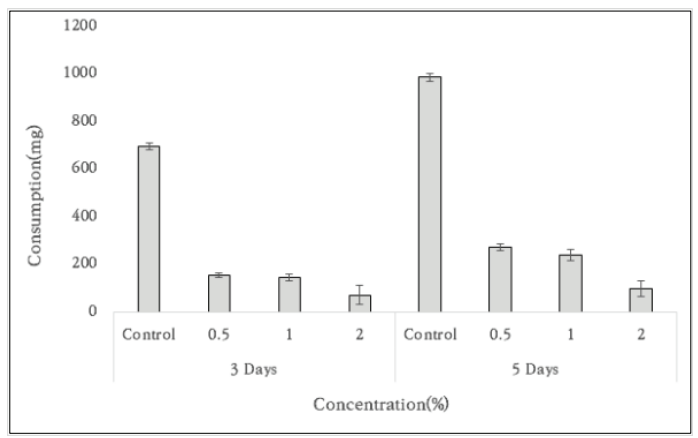

Fig. 2. Feed consumption according to the concentration of the dye marker.

Table 1. Feed consumption ratio to control according to the concentration of the dye marker

\begin{tabular}{ccc}
\hline \multirow{2}{*}{$\begin{array}{c}\text { dye marker } \\
\text { concentration (\%) }\end{array}$} & \multicolumn{2}{c}{ Consumption ratio to control (\%) } \\
\cline { 2 - 3 } & 3 days exposure & 5 days exposure \\
\hline \hline 0.5 & $22.29 \pm 1.20$ & $27.80 \pm 1.26$ \\
1.0 & $20.92 \pm 1.97$ & $24.34 \pm 2.32$ \\
2.0 & $10.52 \pm 5.76$ & $10.17 \pm 3.36$ \\
\hline
\end{tabular}

$0.5 \%, 1 \%$, about $20 \%$ to $25 \%$, and $2 \%$, about $10 \%$. The results showed consistency in feeding trends by concentration, although the overall feeding volume varies with the increasing consumption period. As the concentration of the dye marker increased, the feed intake tended to decrease. This was the same trend in both the 3-day feeding period and the 5-day feeding period. This fact suggests that Sudan Red 7B inhibits the termites' feeding activity.

When Reticulitermes speratus were fed 2\% Sudan Red 7B marked food, as shown in Table 1, feed consumption ratios of $9.6 \%$ (for 3 days) and $10.0 \%$ (for 5 days) were shown compared to the control group. The findings of Grace and Abdallay (1989) were significantly different from our findings. Reticulitermes flavipes were fed with $2 \%$ Sudan Red 7B-marked feed, which showed a feed consumption of about $43 \%$ (for 3 days) and $66 \%$ (for 5 days) compared to the control group. It was found that the feeding behavior was very different between Reticulitermes speratus and Reticulitermes flavipes.

\subsection{Survival rate according to the concentration of the dye marker}

The survival rate of termites exposed to the general food environment after feeding food containing Sudan Red 7B as a dye marker was investigated. The results of the survival rate according to the concentration of the dye marker $(0.5 \%, 1.0 \%$, and $2.0 \%)$ and feeding period ( 3 days and 5 days) are shown in Figs. 3 and 4.

The termites fed the food containing Sudan Red 7B for 3 days showed a survival rate of $90 \%$ or more for 10 days even under the $2 \%$ condition with the highest dye marker concentration. However, for termites

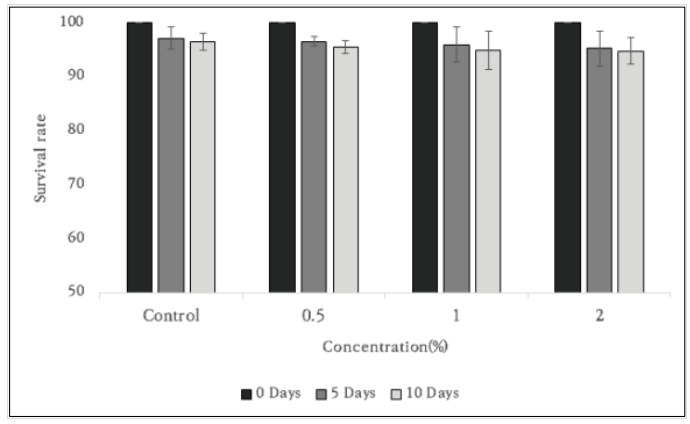

Fig. 3. Survival rate of 3-day feeding termites according to the concentration of the dye marker.

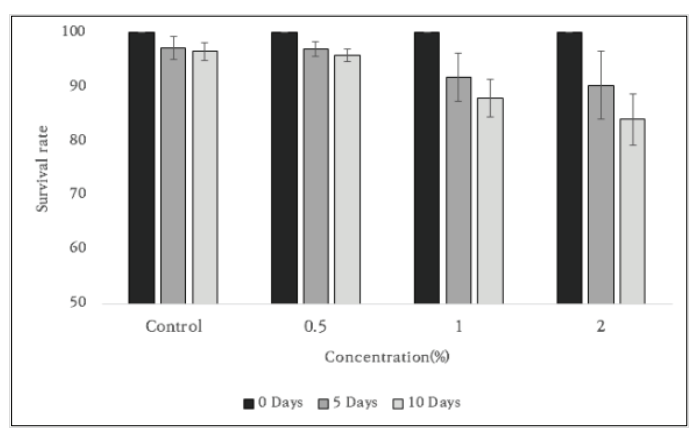

Fig. 4. Survival rate of 5-day feeding termites according to the concentration of the dye marker. 
fed for 5 days, there was no significant difference between the $0.5 \%$ concentration condition group and the control group, but at $1 \%$ and $2 \%$ concentration conditions, the survival rate for 10 days was less than $90 \%$. As the concentration of the dye marker increases or the feeding period of the food containing the dye marker increases, the intake amount of the dye marker increases, and it is considered that Sudan Red 7B has an effect on the survival rate of termites. Grace and Abdallay (1989) reported that when the concentration of $4 \%$ was applied to R. Flavipes, it was difficult to use as a result of a near $60 \%$ mortality rate, and when the concentration of $2 \%$ was fed up to 15 days, the mortality rate was confirmed to be $43 \%$ but could be used. It is also consistent with a study by Grace and Abdallay (1989), who mentioned that a $2 \%$ concentration, approximately $16 \%$ mortality rate in the 5-day feeding condition was confirmed and applicable. As a result of visual observation, it was confirmed that termites that ate food with a dye marker concentration of $1 \%$ or more for 5 days were less active than termites that ate food (all concentration conditions) for 3 days.

In addition, as the concentration of the dye marker increased, the standard deviation of the survival rate tended to equally increase regardless of the feeding period. This is believed to be due to differences in the amount of food intake for each termite individual as the concentration of the dye marker increases. Grace and Abdallay (1989) reported that Sudan Red 7B did not have immediate toxicity to Reticulitermes flavipes for 3-5 days, but showed significant toxicity when exposed for more than 15 days. Delaplane (1988) found that $C$. formosanus workers dyed with Sudan Red 7B had fewer intestinal protozoans and continued to feed less than undyed workers, suggested that the reduced feeding may be due to sublethal physiological effects rather than behavioral avoidance. Therefore, the results of this study are likely to be the same.

\subsection{Retention time of dye marker}

In order to confirm the marking effect that can be monitored for the marked termite individuals, the retention time of the dye marker was checked for the marked termites. The termites were exposed under No-Choice conditions for 3 days and 5 days according to the concentration of the dye marker, so that the termites were stained with the dye marker. Fig. 5 shows the change in body color of termites fed for 5 days with feed containing the dye marker. As shown in the figure, the termite's head turned red. This means that the Sudan Red 7B does not stay in the termite's gut, but spreads throughout the body.

In order to clearly distinguish between marked termites and unmarked termites, termites fed food containing $2 \%$ concentration of the dye marker were studied. As time elapsed as they began to feed on general food, the body color of termites gradually became lighter as shown in Fig. 6. In the case of termites with a feeding period of 3 days, it was difficult to

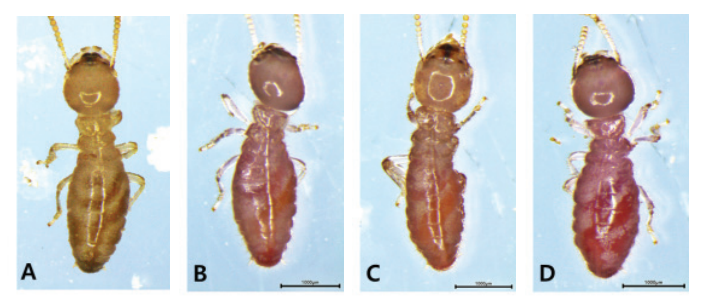

Fig. 5. Marking pattern after 5 days feeding activity according to the concentration of dye marker (A: control, B: $0.5 \%$ C: $1.0 \%$, D: $2.0 \%$ ).

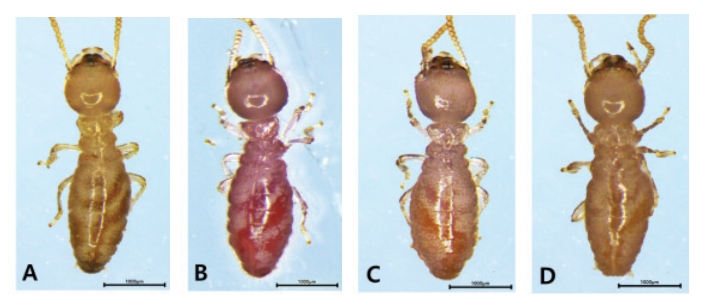

Fig. 6. Changes in body color of marked ( $2 \%$ concentration) termites (A: control, B: after 1-day C: after 4-day, D: after 7-day). 

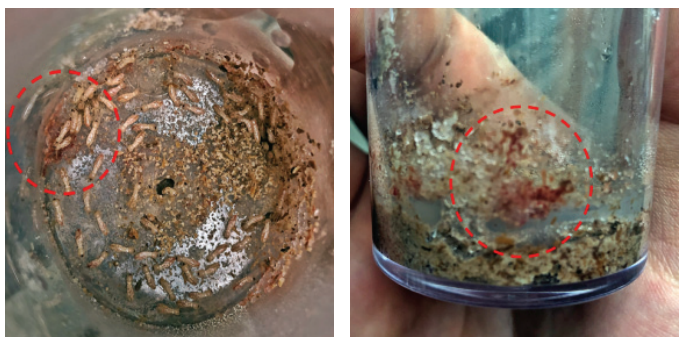

Fig. 7. The images of attachment of termite saliva (red vomit) to the wall of the feeding activity vial.

distinguish them from termites of the control group through visual observation from the elapse of 4 days. In the case of termites with a feeding period of 5 days, it became difficult to distinguish them from termites in the control group after 10 days. However, in the process of feeding general food, the attachment of termite saliva (red vomit) to the wall of the feeding activity vial was observed as shown in Fig. 7. It means that the dye marker in the body of termites are continuously released over time.

As a result of the study, even if feed containing $2 \%$ concentration of dye marker was fed, the duration of the marking effect was only 7 days. In reality, the marking effect is expected to disappear more rapidly because termites feed on a wider variety of food sources than in laboratory conditions. Therefore, in order to estimate the active area of termites using Sudan Red 7B as a dye marker, the moving radius of the marked termites must be confirmed within 5 days from the date of release of the marked termites.

\subsection{Transfer of dye marker}

The same colony, it was confirmed whether staining transfer by the dye marker occurred between the marked and unmarked individuals. As a result of evaluating the transfer effect of the dye marker for termites fed food containing $2 \%$ concentration of the dye marker, the ratio of the number of marked and

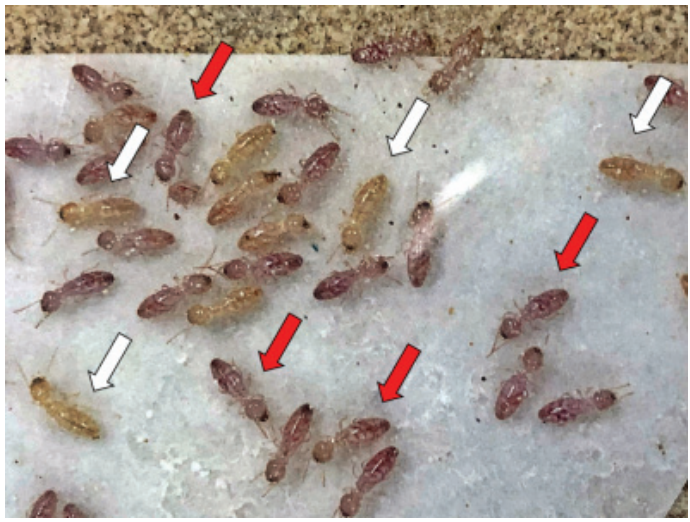

Fig. 8. Marked (red arrow) and unmarked (white arrow) termites.

unmarked individuals was equal to $1: 1$ even after 48 hours. Grace and Abdallay (1989) reported the absence of any appreciable dye transfer by trophallaxis in Reticulitermes.flavipes. In conclusion, it was found that individuals marked with Sudan Red 7B did not transfer the dye marker by trophallaxis (nutrient exchange) to other individuals in the same colony.

\section{CONCLUSION}

Sudan Red 7B was applied as a dye marker in order to find an effective and appropriate marking method to search the territory of the termite (Reticulitermes speratus) colony living in Korea. The sensitivity of termites to the dye marker was evaluated by measuring the feeding amount, survival rate, retention time and transfer efficiency of Reticulitermes speratus according to the concentration and feeding period of Sudan Red 7B. As a result, the following conclusions were obtained.

When Sudan Red 7B was added as a dye marker to the feed, the feed consumption of termites was remarkably reduced, and when the concentration of the dye marker was increased to $2 \%$, feeding disorders were more pronounced. Nevertheless, the survival rate 
of termites was not significantly different from that of the control group as the survival rate of termites was more than $90 \%$, except for the conditions in which feeds containing $1 \%$ and $2 \%$ concentrations of dye marker were fed for 5 days.

In the preceding paper, which evaluated the retention time of marking by feeding the filter paper after marking, it was reported that the unit marked for 5 days with a concentration condition of $2 \%$ was sustained for up to 15 days (Grace and Abdallay, 1989). However, it was found that the $2 \%$ concentration and 5 day condition of the general food (saw dust diet) lasted only about 7 days in this study.

In conclusion, the marking effect of Sudan Red 7B on Reticulitermes speratus was confirmed. Sudan Red 7B was indicated to have no transfer efficiency and survival rate of $2 \%$ concentration condition about $85 \%$ to $R$. speratus. However, when the saw dust diet is fed, the 7 day marking retention time has been confirmed and it is considered that it can be applied as a dye marker to a relatively short-term experiment within 7 days. whether Sudan Red 7B can exhibit sufficient marking effect even in outdoor conditions should be revealed through additional studies.

The laboratory evaluation method for dye markers established through this study will be applicable to various colored dye markers in the future. In addition, it may be usefully used to establish the type and concentration standard of the dye marker applicable to Reticulitermes speratus living in Korea.

\section{REFERENCES}

Atkinson, T.H., Griffin, A.J., King, E.J. 2004. Laboratory and studies of a purple dye marker for Reticulitermes spp. (Isoptera: Rhinotermitidae). Sociobiology 43(2): 259-267.

Atkinson, T.H. 2000. Use of dyed matrix in bait station for determinig foraging territory of subterranean termites (Isoptera: Rhinotermitidae: Reticulitermes spp. and Termitidae: Amitermes wheeleri). Sociobiology 36(1): 149-167.

Chung, Y.J., Kim, S.H., Kim, Y.J., Yu, J.S. 2015. Applicability study on Reticulitermes speratus kyushuensis (Isoptera: Rhinotermitidae) colony eliminator to preserve wooden cultural heritage. Journal of the Korean Wood Science and Technology 43(6): 818-825.

Cultural Heritage Administration. 2014. Manual for cultural heritage care project operation, cultural heritage administration. Incorporated association Cultural Heritage Prevention and Management Center.

Cultural Properties Committee. 2019. Minutes of the second subcommittee on architectural cultural heritage, Cultural Heritage Administration, pp. 41-42.

Delaplane, K.S., Bourg, L.A., La Fage, J.P. 1988. Suppression of termite feeding by Sudan Red 7B. Inter. Res. Group on wood preserv. Doc. No. IRG/WP/1344: 4.

Grace, J.K., Abdallay, A. 1989. Evaluation of the dye marker Sudan Red 7 B with Reticulitermes flavipes (Isoptera: Rhinotermitidae). Sociobiology 15(1): 71-77.

Grace, J.K., Abdallay, A. 1990. A short-term dye for marking eastern subterranean termites (Reticulitermes flavipes Koll.) (Isoptera, Rhinotermitidae). Journal of Applied Entomology 109(1-5): 71-75.

Grace, J.K., Abdallay, A., Farr, K.R. 1989. Eastern subterranean termite (Isoptera, Rhinotermitidae) foraging territories and populations in Toronto. The Canadian Entomologist 121(7): 551-556.

Hwang, W.G., Lee, D.H. 2010. A study on the formation and shape of Reticulitermes speratus Tunnel. 2010 Proceedings of the Korean Society 
Laboratory Evaluation of the Marking Effect of Sudan Red 7B on Subterranean Termites (Reticulitermes speratus) in Republic of Korea

of Wood Science and Technology, pp. 281-282.

Judd, T.M., Fasnacht, M.P. 2007. Distribution of micronutrients in social insects: A Test in the Termite Reticulitermes flavipes (Isoptera: Rhinotermitidae) and the Ant Myrmica punctiventris (Hymenoptera: Formicidae). Annals of the Entomological Society of America 100(6): 893-899

Kim, C.G., Han, G.S. 2019. Analysis of Monitoring of Wooden Cultural Properties in Chungcheongbuk-do. 2019 Proceedings of the Korean Society of Wood Science and Technology, pp. 81.

Kim, S.H., Park, C.M., Lee, H.N. 2017. Safety monitoring of important wooden cultural properties. 2017 Proceedings of the Korean Society of Wood Science and Technology, pp. 102.

King, E.G.JR., Spink, W.T. 1969. Foraging galleries of the formosan subterranean termite, Coptotermes Formosanus, in Louisiana. Annals of the Entomological Society of America 62(3): 536-542.

National Research Institute of Cultural Heritage. 2020. Investigation on biological species harmful to wooden cultural heritage. National Research Institute of Cultural Heritage.

Nobre, T., Nunes, L., Bignell, D.E. 2007. Estimation of foraging territories of reticulitermes grassei through mark-release-recapture. Entomologia Experimentalis Et Applicata 123(2): 119-128.

Raina, A., Park, Y.I., Gelman, D. 2008. Molting in workers of the formosan subterranean termite Coptotermes formosanus. Journal of Insect Physiology 54(1): 155-161.

Sattar, A., Salihah, Z., Naeem, R., Farid, A. 2007. Toxicity and retention of dye markers to Heterotermes indicola. Suranaree Journal of Science and Technology 14(4): 385-390.

Son, D.W., Lee, D.H. 2007. Deterioration diagnosis of wooden building damaged by termites. 2007
Proceedings of the Korean Society of Wood Science and Technology, pp. 89-90

Son, D.W., Lee, D.H. 2008. Evaluation on termite damage of the traditional wooden building by non-destructive methods. Journal of the Korean Wood Science and Technology 36(1): 21-29.

Su, N.Y., La Fage, J.P., Esenther, G.R. 1983. Effects of a dye, Sudan Red 7B, on the formosan subterranean termite, Coptotermes formosanus Shiraki (Isoptera:Rhinotermitidae). Material und Organismen 18(2): 127-133.

Su, N.Y., Scheffrahn, R.H. 1986. A method to access, trap, and monitor field populations of the formosan subterranean termite (Isoptera: Rhinotermitidae) in the urban environment. Sociobiology 12(2): 299304.

Su, N.Y., Scheffrahn, R.H. 1988. Foraging population and territory of the formosan subterranean termite (Isoptera: Rhinotermitidae) in an urban environment. Sociobiology 14(2): 353-359.

Su, N.Y., Ban, P.M., Scheffrahn, R.H. 1991. Evaluation of twelve dye markers for populations studies of the Eastern and formosan subterranean termites (Isoptera: Rhinotermitidae). Sociobiology 19: 349 362.

Su, N.Y., Ban, P.M., Scheffrahn, R.H. 1993. Foraging populations and territories of the Eastern subterranean termite (Isoptera: Rhinotermitidae) in Southeastern Florida. Environment Entomology 22(5): 1113-1117.

Tamashiro, M., Fuji, J.K., Lai, P.Y. 1973. A simple method to observe, trap, and prepare large numbers of subterranean termites for laboratory and field experiments. Environment Entomology 2(4): 721722.

Thorne, B.L., Russek-Coen, E., Forschler, B.T., Breisch, N.L., Traniello, J.F.A. 1996. Evaluation 
of mark-release-recapture methods for estimating forager population size of subterranean termite (Isoptera: Rhinotermitidae) colonies. Environment Entomology 25(5): 938-951.

Tsunoda, K., Matsuoka, H., Yoshimura, T., 1998. Colony elimination of Reticulitermes speratus (Isoptera: Rhinotermitidae) by bait application and the effect on foraging territory. Journal of economic Entomology 91(6): 1383-1386.
Tsunoda, K., Matsuoka, H., Yoshimura, T., Tokoro, M. 1999. Foraging populations and territories of Reticulitermes speratus (Isoptera: Rhinotermitidae). Journal of Economic Entomology 92(3): 604-609.

Xing, L., Chouvenc, T., Su, N.Y. 2013. Molting process in the formosan subterranean Termite (Isoptera: Rhinotermitidae). Entomological Society of America 106(5): 619-625. 